\title{
Netilmicin: new approach to treating gonorrhoea
}

\author{
J SÖLTZ-SZÖTS, $\ddagger$ W KOPP, $\dagger$ W GEBHART,* $\ddagger$ J THURNER,* AND CH POITSCHEK $\ddagger$ \\ From the *Ludwig Boltzmann Institute for Research into Infectious Venerodermatological Diseases, the \\ $\dagger$ Public Health Centre, and the $\ddagger$ Department of Dermatology II, University of Vienna, Vienna, Austria
}

SUMMARY Resistance and side effects to antibiotics or other chemotherapeutic agents are the main limiting factors in the treatment of gonorrhoea. As no such side effects have been reported with a new aminoglycoside, netilmicin, the therapeutic efficiency of this substance was evaluated in 1200 patients (690 women, 510 men) with uncomplicated gonorrhoea, who each received a single dose of $300 \mathrm{mg}$ netilmicin by intramuscular injection. Efficacy of treatment was assessed by smear microscopy and culture, which gave negative results in all 1200 patients. No serious side effects were reported.

These results indicate that netilmicin is an extraordinarily safe and efficient agent for treating uncomplicated gonorrhoea.

\section{Introduction}

The development of resistance, treatment failures, and side effects, particularly allergic and toxic reactions to antibiotics and chemotherapeutic agents, account for the major problems in treating gonorrhoea.

It has been estimated that resistance of gonococci to penicillin can be expected in more than 40 countries in the world. ${ }^{1}$ In Europe increasing numbers of penicillinase producing Neisseria gonorrhoeae (PPNG) strains have been observed. In addition, some PPNG strains have shown resistance to spectinomycin, which possibly indicates a new trend in the pattern of resistance of gonoccoci. ${ }^{2-6}$ Another disadvantage of penicillins is a relatively high rate of allergic reactions, which might be life threatening or even fatal in some cases. ${ }^{7-10}$

Because of their high efficacy against gonorrhoea, especially that caused by PPNG strains, cephalosporins are used more and more often. Studies in large population groups, however, show that $8.1 \%$ of patients hypersensitive to penicillins and $1.3 \%$ to $1.9 \%$ of those not hypersensitive show allergic reactions to cephalosporins. ${ }^{7911}$ The use of cephalosporins has therefore been restricted in patients with hypersensitivity to penicillin. ${ }^{11-14}$ The German Federal Health Department (BGA) recommends the use of third generation cephalosporins to treat only severe life threatening infections, such as peritonitis, septicaemia, or meningitis. ${ }^{15}$ Drugs such as ceftriaxone and cefoxtamine are banned for treating gonorrhoea.

Other antibiotics are recommended by the World

Address for reprints: Professor J Söltz-Szöts, Ludwig Boltzmann Institute for Research into Infectious Venerodermatological Diseases, Alserstrasse 4, A-1090 Vienna, Austria.

Accepted for publication 5 August 1986.
Health Organisation (WHO) and the International Union for Investigation of Venereal Disease and Treponematoses (IUVDT) as second line treatment for gonorrhoea (Luger A, unpublished observation).

About $5 \%$ of patients treated with ampicillin or amoxycillin show allergic reactions. In one study sulphamethoxazole-trimethoprim caused allergic side effects in $5.9 \%$ of the patients treated. ${ }^{9}$ The same is true of probenecid, a derivate of the sulphonamides, which is used concomitantly with penicillins or cephalosporins to reduce the renal excretion of these antibiotics. Complications caused by sulphonamides, which are not usually related to the dosage, manifest themselves as haemorrhagic exanthema, vasculitis, erythema exsudativum multiforme, or, at the worst, as Lyell's syndrome. These complications restrict substantially the indications for these antibiotics . Tetracyclines given as tetracycline hydrochloride (500 mg four times a day for one week) or doxycycline (100 mg twice a day for one week) have the advantage that they cure concurrent chlamydial infection. On the other hand high doses and use for prolonged periods often cause gastrointestinal side effects. Spectinomycin is tolerated well most of the time, but is contraindicated in patients with renal insufficiency.

As most of the drugs used have disadvantages, we looked for other possible treatments for gonorrhoea. The following factors influenced the choice of such a new drug: the cure rate of any drug used to treat gonorrhoea should be not less than $95 \%$, it should be efficient against gonorrhoea caused by PPNG strains, should not induce resistance, and should also be effective against pharyngeal gonorrhoea.

As we had had good results in a pilot study with a new third generation aminoglycoside, netilmicin, in treating uncomplicated cases of gonorrhoea, ${ }^{16}$ we 
decided to continue that study. Netilmicin is a semisynthetic aminoglycoside derived from gentamycin $\mathrm{Cla}$. It is highly bactericidal, and its range includes all Gram negative bacteria and Staphylococcus spp. Its use is contraindicated in patients with severe renal damage or allergy to aminoglycosides. Its safety for use in pregnancy has not yet been established.

\section{Patients and methods}

The minimum inhibitory concentration (MIC) of netilmicin against 100 strains of $N$ gonorrhoeae was tested on the correlated concentrations of the drug. According to these concentrations a single dose of 300 mg netilmicin seemed to be appropriate for adult patients. Treatment therefore consisted of a single intramuscular injection of $300 \mathrm{mg}$ netilmicin.

During the four years 1982-5 1200 consecutive patients (690 women $(57.5 \%)$ and 510 men $(42.5 \%)$ ) with uncomplicated gonorrhoea entered the study. Those with pharyngeal gonorrhoea were excluded, as a previous study had indicated high failure rates with netilmicin as single dose treatment. Diagnosis was established by direct smear microscopy and confirmed by culture on modified Thayer-Martin and New York City media. Gonococci were identified by positive oxidase reaction, Gram stain, and sugar fermentation. Material for examination was taken from the cervix, urethra, and rectum of women patients. We examined the urethral discharge of the men and took smears from the rectum, if indicated. Follow up consisted of repeat smears and cultures within seven days of treatment. As the patients were under the weekly control of the Viennese public health officer, there were no defaulters.

\section{Results}

The bateriological evaluation of the 100 strains tested showed a median MIC of $3.57 \mathrm{mg} / \mathrm{l}$ (table). Nine of these strains were PPNG.

TABLE Minimum inhibitory concentration (MIC) of netilmicin for 100 strains of Neisseria gonorrhoeae*

\begin{tabular}{ll}
\hline No of strains & MIC $(\mathrm{mg} / \mathrm{l})$ \\
\hline 1 & 0.78 \\
16 & 1.56 \\
64 & 3.125 \\
17 & 6.25 \\
2 & 12.50 \\
Median MIC & 3.57 \\
\hline
\end{tabular}

*Nine penicillinase producing strains of $\mathrm{N}$ gonorrhoeae (PPNG), one strain resistant to rifampicin.
Injections of $300 \mathrm{mg}$ netilmicin in patients with a median weight of $80 \mathrm{~kg}$ resulted in maximum peak serum concentrations of $14 \mathrm{mg} / \mathrm{l}$ after 45 minutes. The mean serum half life of Netilmicin was two hours, 70$80 \%$ of the active drug being excreted in the urine after 24 hours.

All patients were cured, no serious side effects were reported, no allergic reactions occurred, and no signs of nephrotoxicity or ototoxicity developed.

\section{Discussion}

According to our test results, with a median MIC of $3.57 \mathrm{mg} / \mathrm{l}$, and a published report of values below $2 \mathrm{mg} / 1,{ }^{17}$ serum concentrations after intramuscular injection of $300 \mathrm{mg}$ netilmicin far exceed the MIC for gonococci. The pattern of susceptibility of the nine PPNG strains to netilmicin was comparable with that of other strains. Though serum concentrations for patients weighing less than $60 \mathrm{~kg}$ may reach the upper limits of safety, toxic effects are unlikely for the following reasons: the serum half life of netilmicin is 1.65 to $2 \cdot 7$ hours for patients with normal renal function; ${ }^{18}$ after single dose treatment $70-90 \%$ of the drug is excreted within 24 hours, and exposure to the substance is therefore minimal.

There are no data about the activity of netilmicin against Treponema pallidum, but it can be concluded that netilmicin is inactive, as gentamicin and sisomicin show no activity. ${ }^{19}$ Netilmicin therefore probably does not influence incubating syphilis. There is also no activity against Chlamydia trachomatis..$^{20}$ One step mutation to resistance to modern aminoglycosides rarely occurs ${ }^{21} 22$

The fear of development of resistant strains, treatment failures, and side effects are the reasons that new compounds are regularly tested for the treatment of gonorrhoea. Apart from spectinomycin, an aminocyclitol antibiotic whose chemical structure is closely related to that of aminoglycosides, aminoglycosides have not been used in central Europe to treat gonorrhoea. The reason for the restricted use of aminoglycosides is concern that resistance may develop by one step mutation, which would have a negative effect on their use in treating systemic infections. Recent evaluations, however, have shown that newly developed aminoglycosides do not have a tendency to cause rapid development of resistance.22 Studies using single dose treatment with an aminoglycoside have not resulted in such a development..$^{23}$

The most common mechanism of bacterial resistance to aminoglycosides is a modification of the drug by enzymes. This results in a blockade of the transport of the antibiotic into the cell. Resistance to aminoglycosides is mainly transferred by plasmids. 
Three major enzymes are known that can inactivate aminoglycosides, but only one, acetyl transferase, can affect netilmicin. ${ }^{24}$ This important difference from other aminoclycosides is one of the reasons for the low risk of rapid development of resistant strains. ${ }^{25}$

Aminoglycosides have been used for single dose treatment of uncomplicated urinary tract infections without inducing a rapid change in resistance patterns. ${ }^{23}$ Our results show that $300 \mathrm{mg}$ netilmicin given as a single injection is an effective treatment for gonorrhoea. Exposure to the drug is low, and the risk of side effects is reduced to a minimum. Resistant strains have not been observed. There is no relevant tendency for the susceptibility pattern to change. It can therefore be concluded that netilmicin is a safe and effective drug for the treatment of uncomplicated gonorrhoea.

\section{References}

1. Anonymous. Current treatments in the control of sexually transmitted diseases. WHO/NDT/80, 1983;433:1.

2. Ashford WA, Potts DW, Adams HJU, et al. Spectinomycin resistant penicillinase-producing Neisseria gonorrhoeae. Lancet 1981; ii:1035-7.

3. Eichmann A. Penicillinresistenz der Gonorrhoe in Europa. Hautart 1983;34:537-8.

4. Perine PL, Schall W,Siegel MS, et al .Evidence for two distinct types of penicillinase producing Neisseria gonorrhoeae. Lancet 1977; ii:993-9.

5. World Health Organisation. Surveillance of Neisseria gonorrhoeae producing $\beta$ tactamase (PPNG). Spectinomycin resistance. Weekly Epidemiological Record 1981;56 (No 20): 158.

6. World Health Organisation. Surveillance of $\beta$-actamaseproducing Neisseria gonorrhoeae (PPNG). Resistance to spectinomycin. Weekly Epidemiological Record 1982;57 (No 17):133-4.

7. Erffmeyer JE. Adverse reactions to penicillin. Ann Allergy 1981;47:289-93.

8. Idsoe $O$, Guthe T, Willcox RR, et al. Nature and extent of penicillin side reactions with particular reference to facilities from: anaphylactic shock. Bull WHO 1968;38:159-88.

9. Patterson $R$, Anderson $J$. Allergic reactions to drugs and biologic agents. JAMA 1983;248:2637-45.
10. Petzold D, Hofmann D, Webelt H. Behandlung der Gonorrhoe in der dermatologischen Praxis. Hautarzt 1982;33:595-7.

11. Vogt H. Kreuzallergie von Cephaloridin, Cephalotin und Penicillin. Hautart 1969;20:407-8.

12. Hofmann H. Neuzeitliche Behandlung der Gonorrhoe. Klinikarzt 1980;9:206-9.

13. Knothe H, Dette FA. Antibiotika in der Klinik. Basel, Switzerland: Aesophus, 1980.

14. Korting HC. Zephalosporin-Allergie und ZephalosporinPenicillin-Kreuzallergie unter besonderer Berücksichtigung für die venerologische Therapie bedeutsamer anaphylaktischer Reaktionen. Hautart 1984;35:225-9.

15. Anonymous. Bundesgesundheitsamt ordnet Anwendungsbeschränkuugen und Waruhinweisse für bestimmte Antibiotika (Cephalosporine) an. Bundesgesundheitsamt (BGA) Pressedienst, 1983;16:1.

16. Söltz-Szöts J, Kopp W, Thurner J, Poitschek CH, Stary A. Kritische Bemerkungen zur Gonorrhoe-Therapie. Netilmicin. Z Hautkr 1985;60:1665-81.

17. Anders CC, Sanders WE, Goering RV. In vitro studies with SCH 21420 and SCH 22591: activity in comparison with six other aminoglycosides and synergy with penicillin against enterococci. Antimicrob Agents Chemother 1978;14:178-84.

18. Michel MF. Mode of administration and dosage. In: Michel MF, ed. Current concepts in aminoglycoside therapy :report of a round table discussion. Rotterdam: Excerpa Medica, $1983 ; 19-24$

19. Kahlmeter G. Netilmicin: clinical pharmacokinetics and aspects on dosage schedules. An overview. Scand J Infect Dis [Suppl] 1980;23:74-81.

20. Thero P. Chlamydia trachomatis in gonococcal and postgonoccocal urethritis. British Journal of Venereal Diseases 1978;54:326-9.

21. Lawson DH .Chairman's concluding remarks. In: Richardson RG, ed. Netilmicin - a recent advance in aminoglycoside therapy. Royal Society of Medicine International Congress and Symposium Series No 50, 1982:203-7.

22. Mouton RP. Resistance. In: Michel MF, ed. Current concepts in aminoglycoside therapy: report of a round table discussion. Rotterdam : Excerpta Medica, 1980;7-13.

23. Stille W. Bedeutung der Einmal-Applikation von Antibiotika bei Zystitis. Antibiotika Spektrum 1983;1:4.

24. Phillips I. Aminoglycosides. Lancet 1982; ii:311.

25. Davies J. Courvalin P. Mechanisms of resistance to aminoglycosides. Am J Med 1977;62:868-72. 